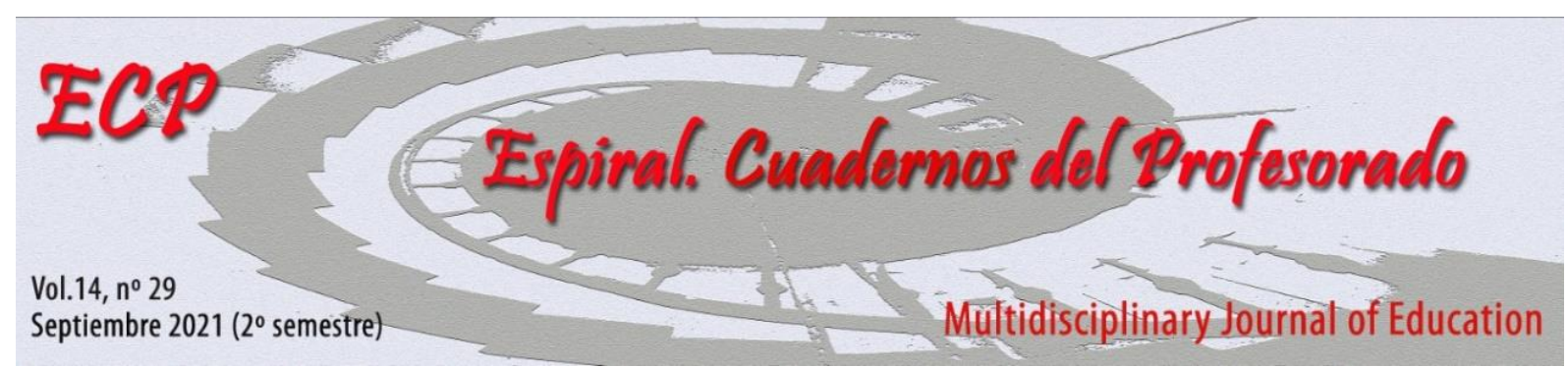

\title{
De la tradición a la realidad 2.0. Análisis semiótico a través de los clásicos en Educación Secundaria
}

\author{
From tradition to reality 2.0. Semiotic analysis through the classics in Secondary \\ Education
}

Pablo Aparicio Durán

${ }^{1}$ Universidad de Granada, España

\section{Resumen}

Este trabajo presenta una reflexión sobre los conceptos de "literacidad crítica" y "multimodalidad" en la presente coyuntura de acusada sofisticación tecnológica y crisis social. La propuesta didáctica, de carácter semióticodiscursivo, trata de ofrecer una sencilla manera para despertar en el alumnado preuniversitario la necesaria capacidad crítica para discriminar tanto las fuentes de sus lecturas como los matices que informan su escritura con elementos que oscilan entre lo subjetivo y lo objetivo, entre lo privado y lo público. En definitiva, los componentes de lo que significa decir "yo soy" en la actual coyuntura histórica. Este último aspecto, sin embargo, no ha sido suficientemente desarrollado en la mayoría de estudios de didáctica, incluso en los de carácter sociológico y etnográfico. De ahí que nuestra propuesta incluya una serie de matizaciones al respecto de los conceptos de "comunicación situada", contexto o "componente cultural".

Palabras clave: Literacidad crítica; Semiótica discursiva; Didáctica de la lengua; Ideología.

\begin{abstract}
This paper looks into the concepts of "critical literacy" and "multimodality" and their implications for both the acute technological sophistication and the present social predicament. The didactic proposal is of a semioticdiscursive nature. It tries to offer a simple way to achieve the necessary critical capacity of Secondary Education students to discriminate both the sources of their readings and the nuances that inform their writing with elements that oscillate between the subjective and the objective, and between the private and the public -- the components of what it means to say "I am" in the current historical conjuncture. This last aspect, however, has not been sufficiently developed in most didactic studies, even those of a sociological and ethnographic nature. Hence, our discussion of the concepts of "situated communication", "context" or the "cultural component".
\end{abstract}

Keywords: critical literacy, discursive semiotics, language teaching, ideology.

Fecha de recepción: 28/01/2021

Fecha de aceptación: 31/07/2021

Correspondencia: Pablo Aparicio Durán, Universidad de Granada, España Email: pabloaparicio@ugr.es 


\section{Introducción: la literacidad crítica}

El primer aspecto que ofrece ya pocas dudas sobre su complejidad para la investigación en Didáctica de la Lengua es el hecho de que el canal comunicativo digital (los dispositivos móviles, el hipertexto y su articulación audiovisual) excede en mucho al tipo de conceptualización eminentemente cognitivista que ha dominado la tematización teórica sobre lectoescritura en los últimos cuarenta años (vid. García Rivera y Martos García et al., 2008). En efecto, la coyuntura económica, política e ideológica exige no solo que la escolarización apunte, ya desde la Educación Infantil, a la consecución de la "competencia comunicativa" (Hymes 1972), sino que, en dicho proceso, se sienten las bases para informar tal competencia con el carácter cualitativo que implica la adquisición de la llamada "literacidad crítica" (Cassany 2015, pp. 90-91).

En definitiva, se trata de lograr que, a través de las etapas de Educación Infantil, Primaria, Secundaria Obligatoria (ESO) y Bachillerato, el estudiantado tenga la oportunidad de trascender realmente (es decir, en la práctica real/social de sus vidas cotidianas) ese estado contradictorio que acusa la tajante división entre las denominadas "prácticas letradas dominantes" (académicas, este caso) y las "vernáculas" (todo ese universo paralelo y desbordante de tipos de lecturas y escrituras en red en el que el individuo -en parte ${ }^{1}$ - se hace a sí mismo). Más aún cuando el ámbito académico ha iniciado, a la vez, su transición, no solo al formato digital, sino a una docencia y a una evaluación también virtuales ( vid. García Peñalvo et al., 2020). Un estado de cosas, pues, en el que -todavía- nos vemos hasta cierto punto alienados e incluso (auto)marginados tantísimos individuos (millennials o no) cuyas destrezas y estrategias letradas adquirimos en una especie de clandestinidad mal entendida (Cassany y Hernández 2008; Aliagas et al., 2009; Zhang y Cassany 2019).

Como decimos, el enfoque cognitivo dominante ha obviado el hecho de que cuando se escribe y se lee, se está participando en una u otra práctica social. El hecho de relegar lo social a lo contextual ha equivalido casi siempre, hasta hace bien poco, a deslindar el aspecto externo de la escritura y la lectura frente a los procesos lingüístico-cognitivos que constituirían su interior. No ha sido hasta la llegada de los Nuevos Estudios de Literacidad (NEL) cuando hemos asistido (sin menoscabo del ingente caudal de conocimientos aportados por el cognitivismo y, hoy, por la neurociencia) a un verdadero cambio de perspectiva (vid. Gee 2005) que cuenta con lo social como parte indisoluble de lo que "significa leer y escribir":

Los Nuevos Estudios de Literacidad (NEL) nacen de una revisión epistemológica lógica de los conceptos tradicionales de lectura y de escritura elaborados desde visiones psicocognitivas. Parten de la intuición de que leer y escribir no es algo ni tan uniforme ni tan solo lingüístico como se había creído. Con una actitud cartesiana se preguntan ¿qué significa leer y escribir? y ¿para quién son buenas estas definiciones socialmente naturalizadas? Y luego salen al mundo para observar y documentar cómo lee y escribe la gente. Con esta proyección empirista se desmarcan de las investigaciones experimentales de laboratorio que han dominado el estudio de la lectura y la escritura. A partir de una mirada etnográfica desarrollan una definición ecológica de estas nociones, tratando de comprenderlas desde la doble dimensión émica y holística que caracteriza a las aproximaciones antropológicas.

Su marco teórico, concebido en la década de los 90, se nutre de la Etnografía de la Comunicación, de las ideas vigotskianas sobre la cognición situada y del debate de la Gran División, que separa lo hablado de lo escrito. Postulan que leer y escribir son actividades humanas específicas, es decir, situadas histórica, social y personalmente en la vida de cada individuo y comunidad, según sus intereses y necesidades. En el mundo, pues, existen formas de leer y de ser lector que son socialmente estructurantes, y cada individuo se las apropia en un proceso de enculturación que es particular, íntimo y creativo. Esta idea les lleva a

\footnotetext{
${ }^{1}$ En un trabajo anterior hemos desarrollado esta matización al respecto de la relativa autonomía del individuo que participa de estas prácticas letradas vernáculas, destacando el carácter ideológico de ciertos presupuestos que operan en los denominados Nuevos Estudios de Literacidad (vid. Aparicio y Martos 2020).
} 
engendrar dos conceptos analíticos que son clave en su formulación teórica y metodológica: el de práctica letrada y el de identidad lectora.

En definitiva, leer y escribir supone todo un ejercicio de (des)codificación de ideas que ha sido bien estudiado. Asimismo, los mecanismos lingüísticos y discursivos que implican la subjetividad o la objetividad han sido de sobra inventariados. Sin embargo, creemos que no se ha hecho suficiente hincapié en la delimitación de la base ideológica sobre la que se asientan dichas ideas, la cual supone toda una variedad de formas y modos de comunicación que implican la oscilación o el estricto posicionamiento del propio discurso entre lo que se considera subjetivo y objetivo (vid. Rodríguez 2015). Esto es importante por una sencilla razón: discurso y sociedad son inseparables; por lo tanto, la subjetividad y la objetividad no pueden analizarse sin tener en cuenta su valor social.

\section{Estado de la cuestión. A vueltas con los conceptos de Multimodalidad y Semiótica discursiva}

Que la comunicación digital ha supuesto el estallido definitivo para el modelo de alfabetización secular y para la comunicación tradicional en general ya nadie lo duda (cfr. Cassany 2011). Sin embargo, no se ha respondido aún a la pregunta de por qué (o por qué no) la "multimodalidad" a la que nos abocan los nuevos medios/modos tecnológicos es prácticamente indescriptible. No obstante, nosotros consideramos que una aclaración sobre cuestiones básicas de crítica ideológica, la cual expondremos a continuación, permite empezar a formar en nuestro alumnado un criterio lo suficientemente informado al respecto, precisamente por recurrir para ello a su experiencia previa. Partimos, para lograrlo, de un simple análisis semiótico-discursivo (cfr. Cotacio 2011) consistente en un ejercicio de "traducción" o "trasvase" entre el discurso literario y el conversacional/digital de WhatsApp. De este modo pretendemos aclarar, si no todas, al menos sí algunas de las cuestiones que consideramos básicas para afianzar el desarrollo de la "literacidad crítica" (Cassany 2019), tan necesaria en un momento, el presente, en el que "leer la ideología" ya no es una tarea para iniciados (id est, continuadores de la Escuela de Frankfurt, los trabajos de Foucault o los de Derrida). Leer la ideología supone, en gran medida, un tipo de lectura que ha sido descartada sistemáticamente: la lectura del "inconsciente ideológico".

Efectivamente, si tenemos en cuenta el hecho de que el lenguaje verbal ha sido (y es) el objeto de estudio por excelencia de la lingüística desde que esta existe y que el resto de elementos expresivos "no verbales" de la comunicación convencional constituyen -todavía- una constelación poco explorada, el análisis de los factores más o menos análogos que se articulan hoy para la comunicación en el universo digital/virtual se torna tanto más complejo. Pero también podemos argüir que lo digital/virtual se limita a reproducir y amplificar (diseminando y homogeneizando su uso) el potencial ya existente en la multimodalidad de toda la vida, y en parte estaríamos en lo cierto: pensemos en una situación en la que dos o más personas reunidas "presencialmente" se comunican a través de elementos discursivos no verbales que acarrean toda una serie de sentidos, conscientes e inconscientes, que van mucho más allá de sus gestos, como son el estilo de ropa, la hora a la que llegan a la reunión, el uso de perfumes o la elección del recinto o sala donde tiene lugar el encuentro.

Traslademos ahora esta simple situación a un contexto interactivo virtual, como puede ser una conversación por WhatsApp en la que los participantes no solo intervienen con mensajes cortos de texto, sino que se intercambian enlaces de interés, fotografías, memes y audios (propios o ajenos) o simplemente se limitan -alguno(s) de ellos, por ejemplo en un "grupo"- a leer los mensajes sin intervenir en la conversación; o directamente a "silenciar" la entrada de mensajes de ese grupo para desentenderse del mismo.

En el caso de los participantes "activos", se producirá un uso determinado de carácter expresivo/afectivo, normalmente- humorístico, haciendo uso para ello del gran repertorio de "emoticonos", algunos de ellos más dependientes del contexto inmediato (el texto escrito) que otros, pero todos, en definitiva, referentes a ideas o nociones solo reconocibles pragmática o situacionalmente: casi siempre -por ejemplo- es posible identificar, de uno u otro modo, el mecanismo retórico de la atenuación e -incluso- la (auto)parodia. Tanto es así que, en su defecto, la intervención de cualquier participante será interpretada, en muchos casos, como un poco "grave" o "seca". En este caso, 
estaríamos hablando de un aspecto pragmático muy fácil de trasladar a una situación dialógica presencial: el tono de voz, la mirada o el gesto de la cara, hablar con las manos en los bolsillos, etc., pueden significar obstáculos a lo que, desde una óptica habermasiana de la comunicación, se ha llamado el "entendimiento".

Cotacio (2011) recoge las siguientes ideas a propósito del texto tradicional (el subrayado es nuestro):

Continuando con las apreciaciones de Lozano y otros (1986), la semiótica debe centrarse en el hacer de los signos más que en lo que representan. Su función es dar cuenta de las formas y estrategias discursivas a través de las cuales enunciadores y enunciatarios se inscriben en un texto y así mismo, concientizar [sic] acerca del itinerario de sentido del mismo. Se abre pues el camino hacia una nueva forma de análisis, ya que el texto así entendido, contiene instrucciones de uso lector, que actúan como huellas del sujeto enunciador así como presuposiciones con respecto a la fase de recepción que consagran estereotipos de enunciatarios y formas concretas de percibir el entorno (Lomas 1997: 2-3)

Pero nos equivocaríamos al considerar estos rasgos como exclusivos del ámbito digital. El acento en lo audiovisual y la necesidad, para todo usuario y sin excepciones, de editar los propios mensajes no le confiere a la actividad discursiva de las redes un sentido distinto al que pudiera tener una situación comunicativa presencial. El sentido del discurso es algo inherente a la sociedad que lo articula de múltiples formas. Ahora bien, en todo momento de su interacción virtual cotidiana, dicho usuario de la Red (con mayúscula) está ejercitando su "competencia semiótica" a un nivel -aquí sí- inusitado en lo que llevamos de modernidad.

\section{Propuesta Didáctica}

Cualquier planteamiento epistemológico de la enseñanza significativa ha de sustentarse en una propuesta empírica válida y contrastada (cfr. Cassany 2016). Pero ¿por dónde empezar? Nuestro propósito aquí es demostrar cómo, desde la Didáctica de la Lengua y la Literatura, se hace imprescindible lograr un grado mayor de "conciencia discursiva". Para ello, vamos a incidir aquí en un aspecto crucial para lograr la consolidación de ese criterio, sin el cual las prácticas letradas (tanto las académicas/dominantes como las vernáculas) no solo no aprovechan, sino que pueden verse excluidas mutuamente por una serie de prejuicios fruto del divorcio entre realidad práctica y realidad teórica.

Dicho aspecto importante es la capacidad o competencia semiótica, que, a nuestro parecer, no solo requiere de una reformulación que la adapte a la era de la comunicación multimodal (Solano 2020), sino que dicha diversidad de formas de participación en el nuevo medio/modo cultural ha de completarse con una mayor capacidad crítica y autocrítica. Significa esto que las destrezas lectoescritoras ya no pueden limitarse a la capacidad expresiva/comprensiva de los mensajes, sino que estos, incluso los propios, deben poder analizarse en su sentido radicalmente histórico.

Pero, ¿qué significa ejercitar? No nos engañemos, el mayor nivel de "ejercicio" de una "competencia" tan solo nos indica que se está produciendo un proceso de "especialización" cultural, y dentro de esta, una variedad de "lenguajes" que se reproducen de una determinada forma que activa unos determinados modos de interpretación, lo cual conlleva siempre un proceso de "cambio" en tanto que el uso de determinados signos. Esto nos lo enseña la sociología y, en concreto, la sociolingüística. Pero en ningún momento estos procesos significan un cambio de sentido en nuestras interacciones Todos ellos son signos que denotan y connotan una serie de (micro)valores expresivos cuya significación está ya de antemano en la lógica social y, por lo tanto, ideológica.

Nosotros sostenemos que tratar todas estas cuestiones como mero componente cultural supone, paradójicamente, desestimar el núcleo del sentido de toda comunicación (vid. Aparicio 2018a). En efecto, el componente cultural se entiende todavía como el marco referencial y atiende, sobre todo, a costumbres y a maneras de ver el mundo en virtud de las cuales los hablantes adoptan una u otra actitud o distancia con la realidad a través de sus expresiones: explícita o implícitamente (cfr. Heidari et. al. 2014). Para nosotros esto no es un componente, sino la base ideológica del discurso. Si entendemos la

Espiral. Cuadernos del Profesorado | ISSN 1988-7701 | 2021, 14(29), 65-72 
ideología como la relación imaginaria de los individuos con sus condiciones reales de existencia, resulta que en todo momento las estamos (re)construyendo -para reproducirlas- mediante el discurso.

Si bien la "comunicación" es todo aquel fenómeno lingüístico acontecido en un contexto, la incógnita la plantean hoy las nuevas condiciones de enunciación: la rápida transformación técnica (las constantemente actualizadas TIC) conlleva una serie de consecuencias en cuanto a los usos y hábitos comunicativos aún por determinar; digamos que el desarrollo tecnológico está aportándole a nuestra sociedad (llamémosla sociedad capitalista/financiera en crisis) una serie de condiciones materiales de reproducción económica, política e ideológica que apenas estamos empezando a calibrar.

Por su parte, la comunicación como concepto general, es el eje de la enseñanza y aprendizaje de lenguas, el cual ha asumido como "norma", al menos a nivel discursivo/público, el llamado "enfoque comunicativo" (y ello ya antes de que fuera institucionalizado por el Marco Común Europeo de Referencia); si bien se habla de lecturas y escrituras "situadas", es decir, contextualizadas funcionalmente en tanto que portadoras de un determinado valor social (UNESCO, 2006), etc., también podemos afirmar que la realidad práctica de la enseñanza se sitúa hoy en un escenario de crisis evidente.

En efecto, la presente coyuntura (de transición económica, política y social -y, por lo tanto, también, ideológica-, unida a los enormes avances en inteligencia artificial) resulta difícil de diagnosticar desde el punto de vista educativo. En lo que concierne a la Didáctica de la Lengua y la Literatura, los retos se resisten a ser descritos concretamente, en primer lugar, porque se carece del lenguaje necesario para hacerlo.

Aterrizando en lo puramente empírico, como ha señalado Elisabeth Melguizo (2020), "es importante que los futuros docentes [de ESO] expliquen a sus alumnos las diferencias existentes entre escritura académica y escritura digital y reconozcan el potencial didáctico que tiene trabajar con WhatsApp, tras las normas establecidas por el Libro de estilo de la lengua española (2018)".

Con todo, nuestro propósito pasa por ir más allá, trasciendo esta dimensión hasta un nivel propiamente semiótico. Nuestra propuesta se materializa en un ejercicio en el que los estudiantes han de transformar un fragmento de texto narrativo (en este caso de novela) en un mensaje de WhatsApp. Tras haberlo hecho espontáneamente, el docente modera un debate sobre los elementos que guían tanto la significación como la situación del texto virtual resultante. Dichos elementos son los anteriormente descritos en el epígrafe 2. Media docena de principios célebres de novelas de la literatura universal nos sirven para hacer una práctica de competencia discursiva en el que no solo nos ejercitamos en el parafraseo, más o menos libre, sino que observamos todos los matices posibles, habida cuenta de la brevedad de ambos textos. Seguidamente (figura 1), ofrecemos el ejemplo de Ana Karenina de Liev. N. Tolstói en su traducción española (2010).

\section{Figura 1}

Fragmento del comienzo de la novela de Tolstói, seguido de una versión coloquial adaptada a la aplicación WhatsApp.

\section{Ejercicio:}

"Todas las familias felices se parecen unas a otras; pero cada familia infeliz tiene un motivo especial para sentirse desgraciada.

En casa de los Oblonsky andaba todo trastrocado. La esposa acababa de enterarse de que su marido mantenía relaciones con la institutriz francesa y se había apresurado a declararle que no podía seguir viviendo con él.

Semejante situación duraba ya tres días y era tan dolorosa para los esposos como para los demás miembros de la familia. Todos, incluso los criados, sentían la íntima impresión de que 


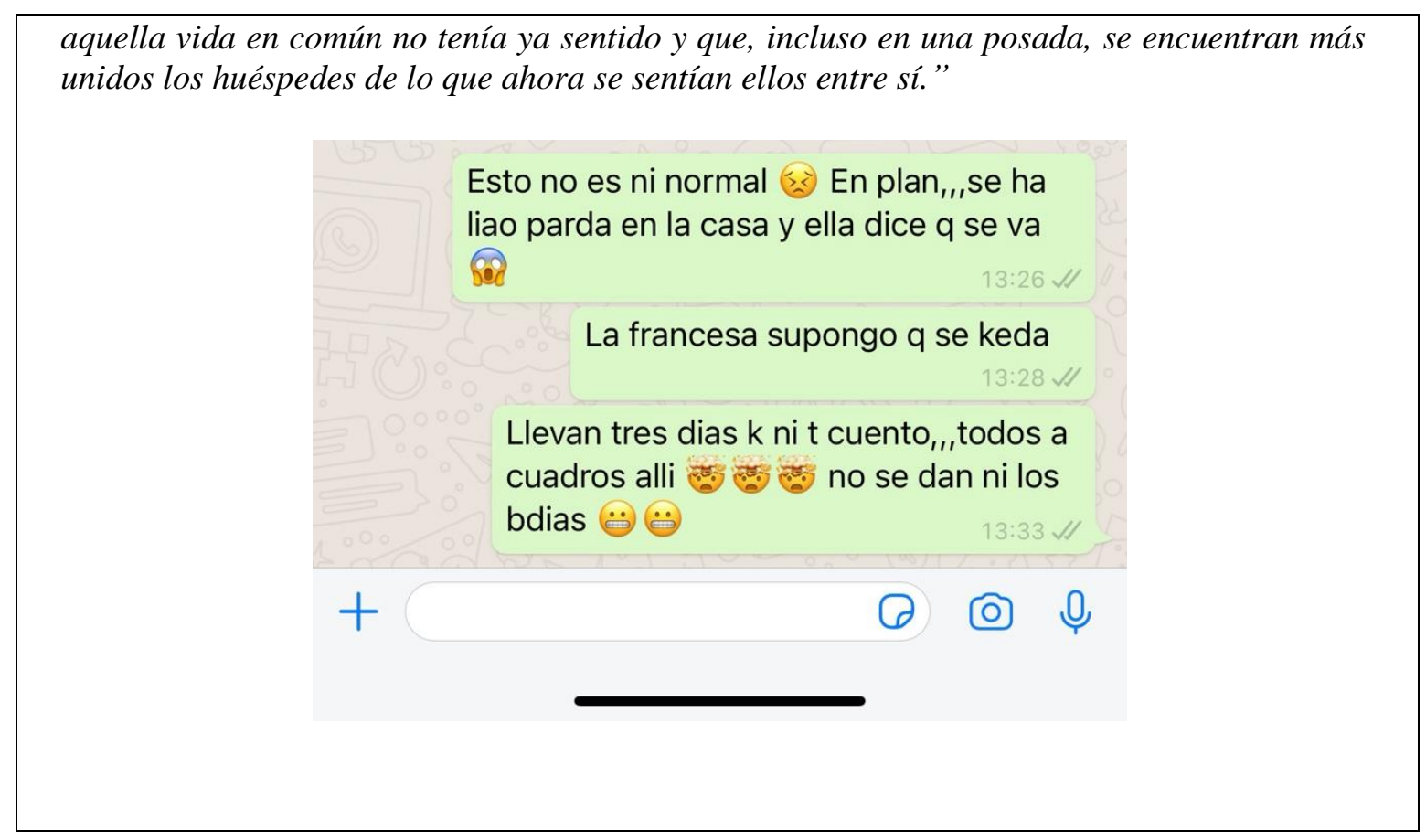

Pero no se trata solo -aunque nuestra propuesta así lo prevea en un principio- de un problema de equivalencias pragmáticas/retóricas, es decir, de una traducción que trasplante la multimodalidad de un discurso a las constricciones o posibilidades del otro, es decir, pasar del discurso -digamos- natural (y sus unidades verbales o no verbales de sentido) al discurso digital (y las suyas), sino que se trata de una cuestión ideológica mucho más sencilla y práctica: el discurso es aquello que hacemos con el lenguaje para construir nuestro propio yo; "construir", aquí, significa legitimar, esto es: inscribirnos, mediante el sentido básico de nuestros enunciados, en una determinada "idea de nosotros mismos en la sociedad", o lo que es lo mismo, una determinada "posición de clase" (eso que en tantas ocasiones se ha entendido erróneamente como una actitud más o menos pretenciosa de superioridad económica, política e ideológica), algo que simplemente se reduce a adoptar una posición respecto de aquello que constituye el núcleo o la norma invariable en cada coyuntura histórica (Rodríguez 2017). Sin tener esto claro, no entenderemos el alcance real de nuestros dispositivos móviles de comunicación (cfr. Agamben 2011)

\section{Conclusiones}

El discurso nos ofrece los síntomas de todo aquello que supone cambios estructurales o sistémicos en cualquier momento. La crítica del discurso, tal como nosotros la concebimos (Aparicio 2018b), es el sismógrafo de la realidad social, parece indicar que el terremoto se está produciendo en el mismo núcleo de nuestras relaciones sociales, esto es, en la matriz ideológica que está en la base de toda interpelación: la noción de sujeto. Si el sujeto comunicativo había sido hasta ahora un sujeto eminentemente lingüístico (ya que el lenguaje era, como digo, hasta hace poco, esa noción que mejor transcribía la contradicción entre la necesidad u obligación de vender cada uno/a su fuerza de trabajo y la necesidad u obligación de hacerlo libremente, hoy este lingüisticismo es multimodal.

La cuestión de los medios o modos no es nueva, de todos es conocida la obra de Marshal McLuhan al respecto: ¿cómo hablar del futuro de la enseñanza sin recurrir a categorías cuyos sentidos están forjados en problemas y necesidades del pasado: ¿qué significan hoy la competencia comunicativa, la motivación, la atención, el gusto por la lectura, el texto y el contexto? Es cierto que son tratadas como categorías universales, sin tener en cuenta que el contenido de esos conceptos depende de los problemas de hoy, que son muy distintos a los de décadas pasadas, a los efectos de considerar conceptos aquí desarrollados como multimodalidad y la necesaria literalidad crítica. 
No obstante, aunque la problemática siga presentando aristas, el consenso en la comunidad educativa parece ser cada vez más significativo: el mundo posmoderno (o "líquido", en palabras de Bauman) en el que vivimos pide a gritos una escuela a la altura de los cambios sociales y tecnológicos que hemos presenciado en estos dos primeros decenios del siglo XXI. De no ser así, nuestro currículo (cfr. Apple 2016), no solo la economía, nos aboca a una situación límite (cfr. Lanier 2011).

Conflicto de Intereses: El autor declara que no tiene conflicto de intereses".

\section{Referencias}

Agamben, G. (2011). ¿Qué es un dispositivo? Sociológica (México), 26(73), 249-264.

Aliagas, C., Lidon, J. M. C. \& Cassany, D. (2009). “Aunque lea poco, yo sé que soy listo”. Estudio de caso sobre un adolescente que no lee literatura. Ocnos: Revista de estudios sobre lectura, (5), 97-112. https://doi.org/10.18239/ocnos_2009.05.07

Aparicio Durán, P. (2018a). Ideología y competencia comunicativa. Fundamentos epistemológicos para la enseñanza de la lengua y la literatura. Visor Libros

Aparicio Durán, P. (2018b). Bases ideológicas del discurso (pos) moderno. Para leer el porvenir de la poesía. En Nuevas poéticas y redes sociales: Joven poesía española en la era digital (pp. 101-112). Siglo XXI.

Aparicio Durán, P., \& Martos García, A. (2020). ¿Son lingüísticas la cultura y la identidad? (Notas al margen sobre el "giro social" en Didáctica de la Lengua y la Literatura). Porta Linguarum. An International and Interuniversity Journal of Foreign Language Teaching and Learning, (34), 193-208. https://doi.org/10.30827/portalin.v0i34.16740

Aliagas, C., Lidon, J. M. C., \& Cassany, D. (2009). “Aunque lea poco, yo sé que soy listo”. Estudio de caso sobre un adolescente que no lee literatura. Ocnos: Revista de estudios sobre lectura, (5), 97-112.

Apple, M. (2016). Ideología y currículo. Akal.

Bauman, Z. (2008). Los retos de la educación en la modernidad líquida. Gedisa.

Cassany, D. (2011). Después de Internet... Textos de didáctica de la lengua y la literatura, (57), 12-22.

Cassany, D. (2016). La escritura extensiva. La enseñanza de la expresión escrita en secundaria. Enunciación, 21(1), 91-106.

Cassany, D., Sala, J., \& Hernández, C. (2008, July). Escribir al margen de la ley: prácticas letradas vernáculas de adolescentes catalanes. In $8^{\circ}$ Congreso de Lingüística General.

Cassany, D. (2015). Literacidad crítica: leer y escribir la ideología. Proyecto Identitades y Culturas Digitales en la Educación Lingüística (ICUDEL15) https://www.researchgate.net/publication/251839730_Literacidad_critica_leer_y_escribir_la_ideologia

Cotacio, M. E. D. (2011). La Semiótica Discursiva: Una teoría para mejorar la lectura y el análisis de textos académicos escritos a nivel universitario. Uni-pluriversidad, 11(1), 97-103.

García Rivera, G. \& Martos García, A. E. (coords.) (2008). Nuevas prácticas de lectura y escritura, ANPE, D.L.

Gee, J. P. (2005). The new literacy studies: From'socially situated'to the work. Situated literacies: Reading and writing in context, 2, 177-194.

Heidari, A., Ketabi, S., \& Zonoobi, R. (2014). The role of culture through the eyes of different approaches to and methods of foreign language teaching. Journal of Intercultural Communication, 34 (6).

Hymes, D. (1972) On Communicative Competence. En Pride, J. B. \& Holmes, J. (Eds.) Sociolinguistics. Penguin Books.

Lanier, J. (2011). You Are Not a Gadget: A Manifesto (Reprint Edition). Vintage.

Rodríguez, J. C. (2015). Para una teoría de la literatura:(40 años de Historia). Marcial Pons.

Rodríguez, J. C. (2017). Teoría e historia de la producción ideológica. Las primeras literaturas burguesas. Akal.

Solano, Carol. (2020). Competencias semióticas para la alfabetización multimodal en la asignatura de español de secundaria en Costa Rica. Innovaciones Educativas, $22 . \quad 162-185$. https://doi.org/10.22458/ie.v22i33.3037.

Tolstói, L. (2020). Ana Karenina. Espasa (Austral). 
UNESCO. (2006) Education for All: A Global Monitoring Report. Chapter 6: "Understandings of Literacy." p. 147-159. http://www.unesco.org/education/GMR2006/full/chapt6_eng.pdf

Zhang, L. T., \& Cassany, D. (2019). El fenómeno «danmu» y la participación mediática: Comprensión intercultural y aprendizaje de lenguas a través de «El Ministerio del Tiempo». Comunicar: Revista Científica de Comunicación y Educación, 27(58), 19-29. https://doi.org/10.3916/C58-2019-02 